\title{
Field Theory in Geography and Stable Structure of Geoformations
}

Rubtsov V.A.

Kazan Federal University, Institute of Management, Economics and Finance, Kazan, 420008, Russia

Gabdrakhmanov N.K.

Kazan Federal University, Institute of Management, Economics and Finance, Kazan, 420008, Russia

Emai:nz9nz@rambler.ru

Mustafin M.R.

Kazan Federal University, Institute of Management, Economics and Finance, Kazan, 420008, Russia

Arzhantseva N.V.

Kazan Federal University, Institute of Language, 420008, Kazan, Russia

Trofimov A.M.

Kazan Federal University, Institute of Management, Economics and Finance, Kazan, 420008, Russia

\section{Doi:10.5901/mjss.2015.v6n3p673}

\begin{abstract}
One of the most topical problems of modern theoretical geography is the development of its fundamental theses. The solution of this task is primarily connected with the generalization and systematization of the concepts of interaction of nature and society which goes on in geographic space and time. The authors of the paper describe the field theory from the perspective of the position of the stable development of geoformations.
\end{abstract}

Keywords: stable development, geofield, heterogeneity, territoriality, geoformation, geoiconics, differentiation, territorial paradigm

\section{Introduction}

The specific feature of the object and subject of geography runs as following: firstly, modern geography studies not the special form of matter movement but the complex of heterogeneous forms: mechanical, physical, chemical, planetary (geological), biological and social; secondly, through the mass of seemingly accidental interactions of these forms of matter movement we might see particular regularities revealing themselves as essentially specific regularities not shrinking to the sum of their constituents $[1,2,3]$. All this allows the authors to make the conclusion that modern geographic "material" is characterized by its natural essential heterogeneity and homogeneity in terms of existence of common specific geographic regularities.

The real geofield is understood as the whole body of geographic substrate with the most significant material geographic relations and interactions. We can give the image of a geofield as affine heterogeneous moving mass in which constant overflows, transfer of substance, dissipation of energy and information, their concentration and diffusion in different points are observed.

It should be noted that the common notion of the term "geographic field" hasn't been singled out. R.R.Denmukhametov pays special attention to this fact [4]. He points out that there exist 3 main approaches to the treatment of geofield in scientific literature: it manifests itself as the sphere formed by the influence of the active center onto the surrounding centers; as the analogies of the physical fields; by means of giving the geographic content to the categories of "space" and "time". It should also be noted that the definition of the geographic field embraces the mentioned above approaches as special cases of the universal approach to the understanding of the geofield.

The geofield is characterized by different kinds of relations, among which we single out spatial relations (relations 
of coexistence of extension) and temporal (relations of movement, duration).

The geographic space is such an aspect of the structure of the geographic field which reflects the aggregate of the relations of coordination and extension of coexisting heterogeneous states (geosituations) of this field. Close connection of the spatial aspect of geofield and the temporal one allows us to mark out not only the spatial and temporal structures of the geofield but the spatial-temporal ones.

The geographic time is the aspect of the geographic field which reflects the aggregate of the relations of coordination and duration of alternating heterogeneous states (geosituations) of the field.

In the basis of the field theory there lie general scientific knowledge about the dialectics of homogeneity and heterogeneity. This notions allow us to explain the existence, functioning and development (evolution) of the geofield and the whole range of geographic laws and principles which have recently appeared in geographic literature $[5,6,7]$. The main principles are the ones of distributed and local heterogeneity.

The geofield is viewed as the distributed heterogeneity, it's an aggregate of affined states (geosituations) interconnected in space and time. The geofield may approximately be compared to the perfect lattice in the points of which there are located relatively stable local inhomogeneities the aggregate of which creates the main grid of geographic intensity differentiating in strength in different points of the field which leads to the movement (changes) in geofield. From the point of view of the notion of homogeneity the inhomogeneities of the geosituation are viewed as local inhomogeneities, i.e. these are such particular different states of the geofield which arise and decay or exist, function and develop bringing out the holdback or giving rise to the appearance, functioning and development of other geosituations.

In accordance with the concept of heterogeneous geofield, which is being developed by the scientists, the characteristic features of objects determine the structure of space-and-time, and vice versa, the space-and-time determines the characteristic features of objects. These notions are reflected in the positional principle [8]. In other words, the geographic object in the geofield differs from a similar object outside the field in terms of its aims, characteristic features and abilities.

Beside the mentioned principles in the development of the geofield there take part the principles of surrounding neigbourhood, the principle of territoriality, the principle of compromise coexistence, etc.

\section{Method}

The methodological and theoretical positions of the formation of spatial-temporal structures are based on the principles of positionalism, regionalism, phenomenology and synergetics. The usage of the latter leads to the identification of spatialtemporal peculiarities of the geostructural formation. These peculiarities in their turn serve as the basis for formalization of the concepts of geostructures and bring us to search for the adequate methods able to give a quantitative and qualitative description to the state, functioning and development of geoformations in the framework of universal concept of objects as a system and system formalisms [9].

The state of geoformations (or geosystems as their formalized analogies) may be described in different ways: verbally, in tables, in diagrams, different formalisms, etc. However, the most general and at the same time constructive concept of the state is reflected in different systems of regions found on the territory. They represent by themselves the basis for geosystems.

There exist two approaches to the investigation of geosystems and the management of the environment: geosituational and geosystemic [10]. While examining the notions of the geosystem and geosituation and drawing parallel lines with physical phenomena we should mention two variants of the description of a real object. The first variant suggests variables $\mathrm{X}_{1} ; \mathrm{X}_{2} ; \ldots ; \mathrm{X}_{\mathrm{n}}$, thus, we conclude that the integral consists of $n$ functional parts. The other variant is that we should examine the indivisible states of the integral $S_{1} ; S_{2} ; \ldots ; S n$ without giving reference to the contribution of each part into the integral. In the first case the description records the organization of a real object, in the second case its dynamics.

Turning to the terms "geosystem" and "geosituation" we might consider that the geosystem reflects the organization of a geoobject, and the geosituation reflects the dynamics of a geoobject. The state of environment may be viewed as a complex and split-level in space and time aggregate of interconnected situations. The geosituation is viewed as a historically developed aggregate of environmental conditions determining the interaction of environmental components. In a general case geosituations arise as the result of deep interaction of affine heterogeneous environmental components. In a particular case geosituations reflect the result of interaction between natural and socioeconomic components in particular part of the geographic space-and-time.

The general scientific concepts of the dialectics of the homogeneous and the heterogeneous in the evolution of the geographic space-and-time serve as the methodological base of the general approach to understanding their dynamic 
connection [11, 12]. According to these concepts the general structure of geographic space is determined by differentiation and heterogeneity distributed in this space. The existence of the latter is connected with heterogeneity and nonidentity in some parameters of the elements of this structure (for example, its subsystems - natural and socioeconomic).

Being formed under the influence of different-scale socio-economic formations the geographic space is initially heterogeneous and has a complex organization. There are a lot of forms of appearance of socio-economic space heterogeneity, not all of them have been investigated to their full extent by the modern science. The socio-economic situation can be viewed as one of the examples of such forms[13].

The conceptual foundation of the socio-economic situation undergoes the stage of its formation, it is being developed by both domestic and foreign scientists, though the number of scholars working on these issues is relatively small $[14,15,16,17]$. Some researchers give just a partial treatment to the notion of "socio-economic situation", they view it as a methodological base to solve the tasks close to the given one [18].

The conceptual foundation of socio-economic situation exists in the framework of the generic geosituational approach. The given circumstance allows us to define the socio-economic situation as the result of interaction, overlapping, intercrossing of affine and split-level factors (demographic, social, economic, ecological, cultural, etc.) acting on a particular territory. From the perspective of the conception of socio-economic situation the state of natural component is considered implicitly, from the point of view of the influence the socio-economic processes have over it. This kind of interaction creates a particular tension of socio-economic processes of the territory, i.e. it forms some state which undergoes continuous changes in space and time.

There exist two concepts explaining the processes and time-and-space localization of socio-economic situations balanced and external [18].

In the framework of the balanced concept the space location of socio-economic situations is viewed as the result of the action of two main social forces - centrifugal and centripetal. The centrifugal force is the joint motion of people, goods, capital, financial and credit assets, etc., and also the competitive relations with other territories. The centripetal force implies the presence of stable territorial socio-economic connections.

The external concept lies in the acceptance of the fact that space localization of socio-economic situation primarily depends on the action of centripetal social forces. The authors of this concept $[19,20]$ single out several types of centripetal forces the action of which is revealed in the formation of territories with the vividly outlined orientation of economics and social sphere, established network of local centers, etc.

Socio-economic situations are in a definite way correlated with the territorial socio-economic systems the states of which can be described with the help of a set of socio-economic situations of the corresponding level and scale. Spaceand-time instability is the fundamental characteristic feature of socio-economic situations. Their existence may last from several days to several years. That is the reason why the boundaries of particular situations are quite movable and they often have "indefinite" character. In most cases the boundaries of stable socio-economic formations - economic zones and regions - don't coincide with the boundaries of spatial distribution of socio-economic situations of the corresponding scale. The research of the spatial-temporal shift and interlinked transition of situations arouses the biggest interest for the researches.

\section{Result}

The scales of socio-economic situations are different. The comprehensive classification of the scales of geosituations hasn't been developed yet. A great number of researchers single out three main scales of geosituations - local (microlevel), regional (mesolevel) and global (macrolevel).

After this classification we might single out the following kinds of socio-economic situations:

1) local scale situations. Territorially they include agglomerations, groups of settlements, particular human settlements, enterprises, infrastructural objects, etc.;

2) regional scale situations. This type of socio-economic situations reflects the state of complex territorial socioeconomic systems at all levels excluding the general planetary one;

- global scale situations. They territorially embrace the whole Globe.

There exist a big number of intermittent scales for socio-economic situations which should be singled out according to the tasks of a particular research. 


\section{Conclusions}

The above mentioned approach formulates the geosituational concept foundation - the managerial aspect of environmental modelling. This conception has a high resolving power. It helps to quite effectively solve extremely complicated geographic tasks which seemed unsolvable before. More than that, it has brought a lot of assistance in ranging geographic tasks; a certain hierarchy and intersubordination have been set.

The effect of the practical realization of the geosituational conception bears no less significance. We might observe noticeable steps in the field of differentiation of environment. Now we can single out stable, unstable, practical parts, risk zones, growth areas on a particular territory, to map them, and also to find attractors on the territory, etc.

Thus, it gives the opportunity to the specialists of corresponding institutions to model the appearance and manifestations, which is an extremely important condition for managerial decision making.

Our multiple presentations at different international forums (primarily International Geographic congresses, International Geographic Union commissions, etc.) reveal a significant interest to the investigation and in some cases to the application of methods and approaches in practical works (countries of the Commonwealth of Independent States such as Armenia, Kazakhstan, Belarus and others; foreign countries such as Belgium, Germany, Italy, Austria, USA).

\section{References}

Gabdrakhmanov N.K., Rubtzov V.A., Shabalina S.A., Rozhko M.V., Kucheryavenko D.Z The role of territorial organization of cities in the touristic attraction of the region on the example of the Republic of Tatarstan // Life Science Journal 2014;11(11), Pages 451-455.

Denmukhametov, R.R., Zjablova, O.V., Shtanchaeva, M.R. Document Development factors of Kazan region recreation area // Life Science Journal 11 (11), 2014, pp. 317-320

Safiullin, L.N., Gafurov, I.R., Shaidullin, R.N., Safiullin, N.Z. Socio-economic development of the region and its historical and cultural heritage // Life Science Journal 11 (6 SPEC. ISSUE), 2014, pp. 400-404

Denmukhametov, R.R. and O.V. Zjablova,. Geodemographic situation in the Republic of Tatarstan. World Applied Sciences Journal. Volume 30, Issue 11, 2014, Pages 1684-1688.

Gabdrakhmanov N.K. and M.V. Rozhko Positioning of Volga Federal District Regions by Demographic Situation Index // World Applied Sciences Journal, Volume 30 Number 6, 2014. - Pages 792-795.

Bagautdinova, N.G., Safiullin, L.N., Badrtdinov, N.N. The role of consumer expenses in ensuring forward dynamics of the Russian economy // Mediterranean Journal of Social Sciences 5 (12), 2014, pp. $43-48$

Gabdrakhmanov, N.K. and V.A. Rubtsov, 2014. Tourist and Recreational Positioning of Tatarstan Republic: Cluster Analysis. World Applied Sciences Journal, 30(Management, Economics, Technology \& Tourism): Pages 202-205.

Mingaleva, Z., Bunakov, O. Innovative ways of using the tourist potential as the basis of territories development // Life Science Journal. Volume 11, Issue 6 SPEC. ISSUE, 2014, Pages 315-317.

Gabdrakhmanov, N.K. and V.A. Rubtzov, 2014. Geodemographic Polarization Processes: Municipal Level (The Case of the Kukmorsky Municipal District of the Republic of Tatarstan). World Applied Sciences Journal, 30(10): Pages 1317-1320.

N.K. Gabdrakhmanov, V.A. Rubtsov. The Objects of Social Infrastructure in the Social Image of the Region Shaping // Procedia - Social and Behavioral Sciences 140 ( 2014 ) 419 - 421

Gabdrakhmanov N.K., Rozhko M.V., \& Kucheryavenko D.Z. Critical and uncritical regions, Advences in Applied Sciences [Online]. 2014, 02, pp 113-116.

Komarova, V.N., Zjablova, O.V., Denmukhametov, R.R. An infrastructure factor in regional competitiveness // Mediterranean Journal of Social Sciences, 5 (18 SPEC. ISSUE), 2014, pp. 355-360

Trofimov, A.,PianovaO. Social-Economucal Situation Analysis [Text] /A.Trofimov, O.Pianova // 10th Europ. Colloq. on Theoretical and Quantitative Geography, Sept. 6-10, 1997. Rostock, Germany. Inst. of Regional Geography, Leipzig, 1997. - P. 86.

Buscema, M. Constraint satisfaction Neural Networks [Text] / M.Buscema. //Shell for Scemata Analysis.Semion Software, 6. Roma, 1994.

Blayo, F. Application of self-organizing maps to the analysis of economic situation [Text] / F.Blayo. //IOP publishing Ltd and Oxford University Press, 1997. - G.:1:1- G.: 1:5.

Trofimov, A.,Kashbrasiev R., PianovaO. Social-economica1 situation concept [Text] / A.Trofimov, R.Kashbrasiev, O.Pianova. // European Regional Science Assotiation, 38th Congress, Vienna, Austria, 28 Aug. - 1 Sept. 1998. Abstracts.Vienna, 1998. - P. 103-104.

Trofimov, A.M., Khamidulin F.G., Sharigin M.D., PianovaO.V. Geosituation analysis in geography as a basis for spatial choice: applications of non-linear dissipation structures and chaos theory. [Text] /A.Trofimov, F.Khamidulin, M.Sharigin , O.Pianova. //CMGS Conference, University of Lisbon, 28th - 29th Angust, 1998.

Thisse, J. - F. Economics matters for geography ... and vice versa [Text] /J.Thisse // Universite de Paris I-Sorbonne and CERAS-ENPC URA..2036-CNRS, 1995.

Fujita, M. Spatial interaction and agglomeration in urban economics [Text] /M.Fujita // ChatterjiM. and Kuenne R.E., eds., New Frontiers in Regional Science. -London, Macmillan, 1990. - P. 184-221.

Arthur, W.B. Increasing retrns and path dependence in the economy [Text] / W.Arthur // Ann. Arbor.,TheUniversityofMichiganPress, 1994. 